\title{
30-Days Readmission after cardiac surgery in elderly patients
}

\author{
Rehab R. Desouki ${ }^{1}$, Heba M Tawfik ${ }^{1}$, Salma M Elsaid ${ }^{1}$, Hamdy A Singab ${ }^{2}$ and Sarah A \\ Hamza $^{1}$ \\ ${ }^{1}$ Geriatric and Gerontology Department, Faculty of Medicine, Ain Shams University. \\ ${ }^{2}$ Cardiovascular \& Thoracic Surgery Department, Faculty of Medicine, Ain Shams University.
}

\begin{abstract}
Background: Readmissions are a common problem in cardiac surgery. As cardiac operations being common major elective operations in older adults and older adults representing an increasing portion of surgical candidates. Data regarding prevalence of readmission and its risk factors in such population is needed.
\end{abstract}

Aim: To assess the frequency and risk factors associated with hospital 30 days readmissions for elderly patients undergoing cardiac operations.

Methods: A prospective observational cohort study was conducted from the first of March, 2017 to the end of March, 2018, where 180 patients aged $\geq 60$ years undergoing elective cardiac operations in Ain Shams university hospital were included. Participants were subjected to full history taking and physical examination. Type of operation and thirty days readmission following surgery were documented.

Results: 30 days readmission rate was $20.2 \%$ (number of readmissions, 36 ). When stratified by procedure type, readmission rates were isolated coronary artery bypass grafting (CABG), 66.7\% $(n=24)$; isolated valve, $8.3 \%(n=3)$; CABG + valve, $25 \%(n=9)$. Baseline patient characteristics associated with readmission included male gender, elevated creatinine, dementia, congestive heart failure and presence of multiple comorbidities " $\geq 3$ ".

Conclusions: 1 of 5 elderly patients undergoing cardiac operations requires readmission within 30 days after surgery. This outcome has significant health and economic implications. Further studies are needed to assess causes of readmission and to determine which readmission strategies are most effective for elderly patients undergoing cardiac surgeries.

Keywords: surgery in Elderly - coronary artery bypasses grafting - Cardiac surgery in elderly

\section{Background}

Hospital readmission rates following surgery are increasingly used as a marker of quality of care. However, studies on readmission rates in surgical patients are limited by the restricted number of procedures they examine, the exclusive age categories that are included, and the lack of distinction of elderly patients from other surgical patients ${ }^{1 .}$ It has been shown that outcomes in surgical patients, including readmission rates, vary significantly by patient age, procedure type, and surgical specialty ${ }^{2}$.

Older adults are an increasing proportion of surgical Corresponding Author: Heba M Tawfik: hmt82@yahoo.com care, greater than $35 \%$ of all inpatient operations being performed in adults 65 years or older, with cardiac operations being one of the common major elective operations in older adults ${ }^{3}$. However, literature on readmissions after cardiac operations is scant and additional data are needed to identify the prevalence, etiology and risk factors of readmissions in order to develop targeted quality initiatives.

The goal of this study was to assess the frequency and risk factors associated with hospital readmissions for elderly patients undergoing cardiac operations one 
month following surgery.

\section{Methods}

Study design: prospective observational cohort study

Setting: Ain Shams University Hospital.

Study group and endpoints: From the first of March, 2017 to the end of March, 2018, one hundred and eighty participants aged $\geq 60$ years undergoing elective cardiac operations in Ain Shams university hospital were recruited. Patients undergoing urgent or emergency operations were excluded.

The study was approved by the ethical committee of the Faculty of Medicine, Ain Shams University. Written consent was obtained from every participant after explanation of the study aim and procedures. All subjects participating in the study were subjected to full history taking and physical examination. Type of operation and thirty days readmission following surgery were documented as well.

Statistical analysis: Statistical presentation and analysis of the present study was conducted, using the mean, standard error and Chi-square tests by SPSS 17 .

\section{Results}

Among the 180 patients who underwent cardiac surgery, mean age was $64.68 \pm 2.95$ years, $23.9 \%(\mathrm{n}=$ 43) were women and $39.4 \%(n=71)$ were smokers either current or ex-smokers (Table 1). Hypertension was present in $81.7 \%(\mathrm{n}=147)$ of patients, $34.4 \%(\mathrm{n}=$ 62) had diabetes mellitus, 33.9\% $(n=61)$ had pulmonary disease or asthma and $29.4 \%(\mathrm{n}=53)$ had previous mvocardial infarction (Table 2 ).

Table 1: Demography of the study population

\begin{tabular}{|c|c|c|c|}
\hline & & Mean / N & SD / \% \\
\hline \multirow[t]{2}{*}{ Gender } & Male & 137 & $76.1 \%$ \\
\hline & Female & 43 & $23.9 \%$ \\
\hline Age & & 64.68 & 2.95 \\
\hline \multirow{4}{*}{$\begin{array}{l}\text { marital } \\
\text { status }\end{array}$} & Married & 116 & $64.4 \%$ \\
\hline & Single & 16 & $8.9 \%$ \\
\hline & Widow & 39 & $21.7 \%$ \\
\hline & Divorced & 9 & $5 \%$ \\
\hline \multirow[t]{5}{*}{ Education } & Illiterate & 10 & $5.6 \%$ \\
\hline & $\begin{array}{c}\text { can read and } \\
\text { write }\end{array}$ & 17 & $9.4 \%$ \\
\hline & $\begin{array}{l}\text { below high } \\
\text { school }\end{array}$ & 34 & $18.9 \%$ \\
\hline & high school & 55 & $30.6 \%$ \\
\hline & $\begin{array}{c}\text { university or } \\
\text { higher }\end{array}$ & 64 & $35.6 \%$ \\
\hline \multirow[t]{2}{*}{ Smoking } & Yes & 71 & $39.4 \%$ \\
\hline & No & 109 & $60.6 \%$ \\
\hline \multirow[t]{2}{*}{ Smoker } & Current & 22 & $31 \%$ \\
\hline & ex-smoker & 49 & $69 \%$ \\
\hline
\end{tabular}

Table (2): preoperative co-morbidity

\begin{tabular}{|lll}
\hline & $\mathbf{N}$ & $\%$ \\
\hline MI & 53 & $29.4 \%$ \\
\hline CHF & 25 & $13.9 \%$ \\
\hline PVD or bypass & 8 & $4.4 \%$ \\
\hline CVA or TIA & 25 & $13.9 \%$ \\
\hline pulmonary ds or asthma & 61 & $33.9 \%$ \\
\hline DM only & 62 & $34.4 \%$ \\
\hline DM \& end organ damage & 32 & $17.8 \%$ \\
\hline renal disease & 14 & $7.8 \%$ \\
\hline mild liver disease & 14 & $7.8 \%$ \\
\hline peptic ulcer & 18 & $10 \%$ \\
\hline non-metastatic cancer & 2 & $1.1 \%$ \\
\hline Dementia & 5 & $2.8 \%$ \\
\hline rheumatic or CT disease & 15 & $8.3 \%$ \\
\hline HIV or AIDS & 2 & $1.1 \%$ \\
\hline Hypertension & 147 & $81.7 \%$ \\
\hline skin ulcer or cellulitis & 7 & $3.9 \%$ \\
\hline Depression & 8 & $4.4 \%$ \\
\hline warfarin use & 11 & $6.1 \%$ \\
\hline & & \\
\hline
\end{tabular}

The most common procedures performed were isolated CABG $(71.7 \%[\mathrm{n}=129])$ and isolated valve operations $(22.2 \%[\mathrm{n}=40])$. Patients exhibited thirty day readmission rate of $20.2 \%(n=36)$ (Table 3$)$. When stratified by procedure type, readmission rates were isolated CABG, $66.7 \%(\mathrm{n}=24)$; isolated valve, $8.3 \%(\mathrm{n}$ $=3) ; \mathrm{CABG}+$ valve, $25 \%(\mathrm{n}=9)$.

Table 4 and 5 presents the significant risk factors for readmission. They included male gender $(\mathrm{p}=0.018)$, congestive heart failure $(\mathrm{p}=0.03)$, elevated creatinine $(\mathrm{p}=0.035)$, dementia $(\mathrm{p}<0.001)$ and presence of multiple comorbidities " $\geq 3$ " ( $(\mathrm{p}=0.002)$.

Table 3: Type of operation and thirty day readmission rate

\begin{tabular}{llll}
\hline & & $\mathrm{N}$ & $\%$ \\
\hline \multirow{2}{*}{ type of } & CABG & 129 & $71.7 \%$ \\
operation & CABG+valve replacement & 11 & $6.1 \%$ \\
& valve replacement & 40 & $22.2 \%$ \\
\hline \multirow{2}{*}{30 day readmission } & 36 & $20.2 \%$ \\
\hline
\end{tabular}


Table 4: Relation between demographic data and 30 days readmission

\begin{tabular}{|c|c|c|c|c|c|c|c|}
\hline \multicolumn{2}{|c|}{ Demographic data } & \multicolumn{4}{|c|}{30 days readmission } & \multicolumn{2}{|c|}{ Chi-Square test of significance } \\
\hline & & \multicolumn{2}{|c|}{ No } & \multicolumn{2}{|c|}{ Yes } & & \\
\hline & & $\mathrm{N}$ & $\%$ & $\mathrm{~N}$ & $\%$ & P-value & Sig. \\
\hline \multirow{2}{*}{ Gender } & male & 79 & $54.86 \%$ & 30 & $83.33 \%$ & \multirow{2}{*}{0.018} & \multirow[t]{2}{*}{$\mathbf{S}$} \\
\hline & female & 65 & $45.14 \%$ & 6 & $16.67 \%$ & & \\
\hline \multirow{2}{*}{ Smoking } & yes & 53 & $36.8 \%$ & 18 & $50 \%$ & \multirow[t]{2}{*}{0.15} & \multirow[t]{2}{*}{ NS } \\
\hline & no & 91 & $63.2 \%$ & 18 & $50 \%$ & & \\
\hline
\end{tabular}

Table 5: Relation between comorbidity and 30 days readmission

\begin{tabular}{|c|c|c|c|c|c|c|}
\hline \multirow[t]{3}{*}{ Comorbidity } & \multicolumn{4}{|c|}{30 days readmission } & \multirow{2}{*}{\multicolumn{2}{|c|}{$\begin{array}{l}\text { Chi-Square test of } \\
\text { significance }\end{array}$}} \\
\hline & \multicolumn{2}{|l|}{ No } & \multicolumn{2}{|c|}{ Yes } & & \\
\hline & $\mathbf{N}$ & $\%$ & $\mathbf{N}$ & $\%$ & P-value & Sig. \\
\hline MI & 40 & $27.8 \%$ & 13 & $36.1 \%$ & 0.3 & NS \\
\hline CHF & 16 & $11.1 \%$ & 9 & $25 \%$ & 0.03 & $\mathbf{S}$ \\
\hline PVD or bypass & 7 & $4.9 \%$ & 1 & $2.8 \%$ & $0.58(\mathbf{F})$ & NS \\
\hline CVA or TIA & 20 & $13.9 \%$ & 5 & $13.9 \%$ & 1 & NS \\
\hline pulmonary ds or asthma & 49 & $34 \%$ & 12 & $33.3 \%$ & 0.9 & NS \\
\hline DM only & 51 & $35.4 \%$ & 11 & $30.6 \%$ & 0.58 & NS \\
\hline DM \& end organ damage & 22 & $15.3 \%$ & 10 & $27.8 \%$ & 0.07 & $\mathbf{N S}$ \\
\hline renal disease & 7 & $4.9 \%$ & 7 & $19.4 \%$ & 0.035 & $\mathbf{S}$ \\
\hline peptic ulcer & 15 & $10.4 \%$ & 3 & $8.3 \%$ & 0.7 & NS \\
\hline Dementia & 1 & $0.7 \%$ & 4 & $11.1 \%$ & $<0.001(\mathrm{~F})$ & HS \\
\hline rheumatic or CT disease & 13 & $9 \%$ & 2 & $5.6 \%$ & 0.5 & NS \\
\hline HIV or AIDS & 1 & $0.7 \%$ & 1 & $2.8 \%$ & $0.3(F)$ & NS \\
\hline hypertension & 114 & $79.2 \%$ & 33 & $91.7 \%$ & 0.08 & NS \\
\hline skin ulcer or cellulitis & 4 & $2.8 \%$ & 3 & $8.3 \%$ & $0.12(F)$ & NS \\
\hline Depression & 7 & $4.9 \%$ & 1 & $2.8 \%$ & $0.58(\mathbf{F})$ & NS \\
\hline$\geq 3$ comorbidities & 89 & 61.8 & 32 & 88.9 & 0.002 & $\mathbf{S}$ \\
\hline$<3$ comorbidities & 55 & 38.2 & 4 & 11.1 & & \\
\hline
\end{tabular}

\section{Discussion}

Although advancements have been made during the past decade in improving outcomes after cardiac operations, readmission rates remain high ranging from $8.3 \%$ to $21.1 \%$ in patients undergoing coronary artery bypass grafting (CABG) operations ${ }^{4}$

In the current prospective cohort of elderly cardiac surgical patients, the thirty days readmission rate was $20.2 \%$.Although a 30 -days time point for examining readmissions has often been criticized as clinically arbitrary, a large, multicenter, prospective cohort of 5059 adult cardiac surgical patients demonstrated that the 30-days period captures most of the readmissions ${ }^{5}$.

Similar findings were found by Price and associates while studying 1205 patients undergoing CABG with thirty days readmission rate of $13 \%$ and the majority readmissions occurred within 1 week of the primary hours of discharge ${ }^{6 .}$

Given the high volume of cardiac operations in older adults and the cost of such interventions, focusing interventions on high-risk populations might make readmission reduction strategies more applicable.

When demographic characteristics were studied, male gender, congestive heart failure, dementia, elevated creatinine and presence of multiple comorbidities " $\geq 3$ " correlated with an increased risk of readmission. Several of the risk factors observed in our analysis have been identified by other investigators and correlate with the most common etiologies for readmission ${ }^{5,6}$.

Individuals with congestive heart failure and elevated serum creatinine are sensitive to fluid management and at higher risk for readmission for volume overload. These preoperative characteristics and its impact on readmission risk require further exploration.

In this study, male gender was found as a risk factor for thirty days readmission rate. On the contrary, female 
gender was detected as a risk factor by studies conducted by Iribarne et al., $\mathbf{2 0 1 4}$ as regarding readmission and Edwards et al., 1998 as regarding postoperative mortality ${ }^{5,7}$

Our analysis has several limitations: First, data are representative of readmission rates at single academic medical center and do not include outcomes at multiple or nonacademic centers. Second, our analysis did not consider the effect of socioeconomic status because this information was not collected. Lastly, our analysis did not consider cause of readmission as it focused on preoperative risk factors rather than postoperative complications.

\section{References}

1-Tsai TC, Joynt KE, Orav EJ, et al. (2013): Variation in surgicalreadmission rates and quality of hospital care. N Engl J Med.; 369(12): 1134-42.

2-Merkow RP, Ju MH, Chung JW, et al. (2015): Underlying reasons associated with hospital readmission following surgery in the United States. JAMA.; 313(5):483-95.

4-Hannan EL, Zhong Y, Lahey SJ, et al. (2011): 30-day readmissions after coronary artery bypass graft surgery in New York State. JACC Cardiovasc Interv.; 4:569-76.

3-Robinson TN, Wu DS, Pointer L, et al. (2013): Simple frailty score predicts post-operative complications across surgical specialties. Am J Surg; 206(4): 544-50.

5-Iribarne A, Helena Chang H, Alexander JH (2014): Readmissions after cardiac surgery: experience of the National Institutes of Health/Canadian Institutes of Health Research Cardiothoracic Surgical Trials Network. Ann Thorac Surg.; 98:1274-80.

6-Price JD, Romeiser JL, Gnerre JM et al. (2013): Risk analysis for readmission after Coronary Artery Bypass Surgery: developing a strategy to reduce readmissions. J Am Coll Surg.; 216(3): 412-9.

7-Edwards FH, Carey JS, Grover FL, et al. (1998): Impact of gender on coronary bypass operative mortality. Ann Thorac Surg.; 66:125-31. 